\title{
General relativity constrains proper times and predicts frozen stars instead of black holes ${ }^{1}$
}

\author{
Zahid Zakir $^{2}$
}

\begin{abstract}
In a static gravitational field an intersection of a worldline by a global hypersurface of simultaneity $\mathrm{t}=$ const gives an invariant constraint relating the proper time of this event by t. Since at any finite $t$ such constrained proper time intervals are less than required for crossing a horizon, general relativity predicts the gravitational freezing of proper times in stars with timelike or null geodesics everywhere. The time dilation stabilizes contracting massive stars by freezing, which is maximal but finite at the centre and the surface is frozen near the gravitational radius. The frozen stars (frozars) slowly defrost due to emissions and external interactions, the internal phase transitions can initiate refreezing with bursts and explosions.
\end{abstract}

PACS: 04.20.Dg; 04.70.-s; 97.60.-s, 98.54.-h

Key words: relativistic stars, black holes, quasars, active galactic nuclei, collapse, horizon, singularities

\section{Content}

Introduction 1

1. The physical coordinates and the global simultaneity in a static field ........................ 2

2. A constraint for the proper times of particles and a falling thin dust shell .................. 3

3. General properties of the gravitationally-frozen stars............................................. 6

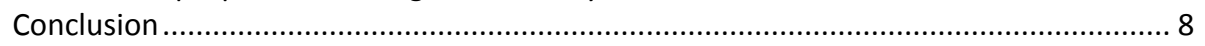

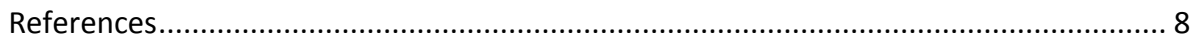

\section{Introduction}

The massive cold stars cannot support themselves against the gravitational contraction. In the Newtonian theory this leads to the quick collapse into the region $r_{g}=2 G M$. In general relativity (GR) the surface of the star becomes frozen near the gravitational radius $r_{g}$ for the distant observers.

But when the surface contracts, a proper time interval $\tau_{g}$, needed to cross $r_{g}$, remains finite and the theory must to interpret this fact. On the basis of this fact the black-hole approach predicted the real black holes (BHs) [1-2]. But one of the basic implicit assumptions of this approach was that the evolution in terms of the proper times can be considered independently from the coordinate time's moments.

Recently in the paper [3] it has been proposed a new treatment of the massive relativistic stars using the fact that in the static fields GR allows one to define a global simultaneity of events in terms of the coordinate time $t$. It has been shown that on the hypersurfaces of simultaneity $t=$ const GR allows one to construct a new

\footnotetext{
${ }^{1}$ The preprint of the paper has been presented in 2007: Zakir Z. arXiv:0705.2585

${ }^{2}$ Centre for Theoretical Physics and Astrophyics, Tashkent Uzbekistan; zahidzakir@theor-phys.org
} 
theory of compact massive stars as the gravitationally frozen objects (frozars) having some internal structure at any moment $t<\infty$.

In the present paper it will be shown that in GR there is an identity $r(t)=r(\tau)$ for the radial coordinate of an event leading to an invariant constraint for the proper times of falling particles $\tau=\tau(t)$ restricting them by relating with $t$ and then it will be discussed some new observable properties of the such frozen stars (frozars) predicting by GR instead of the black holes.

\section{The physical coordinates and the global simultaneity in a static field}

In a rest frame of a centrally-symmetric source the spherical symmetry leads to three key physical properties of the gravitational field around that source:

(1) it is static;

(2) it does not change the length of a perpendicular to radius standard rods;

(3) there is a global simultaneity of events on the hypersurfaces $t=c o n s t$ defined via a set of coordinate clocks synchronized by distant observer's clocks.

In most of coordinate systems of various frames of reference these physical properties become hidden due to insufficient geometrical or kinematical complications. The curvature coordinates $(t, r, \theta, \varphi)$ of a static frame, defined on the hypersurfaces of simultaneity $t=$ const, in a simplest and physically convenient form express these physical properties and the symmetries of the static field. In terms of the curvature coordinates the metric outside and on the surface of a spherical body $r \geq R$ (here $R>r_{g}$ ) is the Schwarzschild vacuum solution, the metric inside the source $(r<R)$, as the matter solution, is regular at any point of the body.

The gravitational time dilation defining by the component of the metric $g_{00}(r)=1-r_{g} / r$ at first leads to the well-known gravitational redshift (blueshift) of outfalling (falling) photons which in the lowest order is the same as in the Newtonian theory.

But the second observable effect, directly showing the time dilation of the standard clocks at comparison with the distant observer's clocks after long term duration in the field, is not so known, but is more important since it is absent in the Newtonian theory. Really, in the Newtonian gravity the rate of a standard clock does not depend on the duration time $t$ in the field. In GR the dilation $\delta \tau$ of a standard clock in the gravitational field with respect to the same clock at the height $h$ is proportional to the duration $t$ and is given by the formula: $\delta \tau=g h t$ (here $g$ is the gravitational acceleration). This integral effect is confirmed in experiments by clocks long-term traveled in airplanes and satellites [4-6]. Thus, further we will follow to Einstein's treatment of proper times as really dilated in the gravitational field (see [6]).

In GR, due to the different rate of the standard clocks at various points, we may introduce an additional set of the coordinate clocks going promptly than neighbor standard clocks so that always be synchronous by distant observer's clocks (see [2]) and at any point showing the coordinate or world time $t$. Therefore, any event at $r$ is labeled not only by the proper time $\tau(r)$, but by the globally defined time $t$ of a nearby coordinate clock. The such defined global hypersurface $t=$ const 
intersects any worldline in the field to three parts containing the events before, simultaneous and after the distant observer's proper time moment $t$. Although at the coordinate transformations the hypersurface can be deformed, nevertheless, the separation of events to these three groups remains unchanged due to the invariant character of the worldlines.

Thus, in GR the relative rates of the standard clocks depend on their location with the absolute dilation of the proper times in the stronger field, and this, experimentally checked [4-6] dependence is invariant fact with which agree all observers. Notice, that the existence in GR of the globally synchronized set of coordinate clocks rejects the arguments about difficulties by the informing of the distant observers since a world time moment $t$ of any event can be determined instantaneously via the nearby coordinate clock. Therefore, if the equations of motion of GR require $t_{g} \rightarrow \infty$ for the crossing $r_{g}$, then this event really occurs when the local coordinate clocks near the source show $t_{g} \rightarrow \infty$.

Notice also that the observers near the massive star detect the violetshift of photons emitted by the distant observer and they are agreeing that distant observer's clocks are going faster than the nearby standard clocks. This means that the time dilation in the gravitational field, as in the case of the twin effect in the accelerated frames, is irreversible and asymmetrical under the observers. This kind of time dilation is in principle differs from the symmetric under the observers and relative time dilation on inertial frames on flat space-time. Thus, the approaches treating the gravitational time dilation as a relative effect are based on a wrong analogy with the time dilation on the inertial frames.

There is also a widely accepted assumption that the curvature coordinates in the static field have not direct physical meaning and can be replaced by more convenient ones. Really, most of arbitrary coordinates contain the "coordinate effects" not describing the properties of the underlying manifold. However, if a coordinate system is constructed as a physical one, i.e. if the coordinates mean the numbers of resting with respect to each other standard rods and the indications of the globally synchronized clocks, then the corresponding metrics describe the physical properties of the manifold.

Since the gravitational field does not change the length of standard rods on 2spheres around the source, the curvature coordinates are such physical coordinates on the hypersurfaces of simultaneity $t=$ const . The corresponding Schwarzschild's metric, therefore, describes true physical properties of the static field. Really, at $r \rightarrow r_{g}$ the proper times really dilate with respect to $t$, the radial rods really contract with respect to the flat case and these effects are experimentally checked. Therefore, the ignoring of the physical character of the curvature coordinates is incompatible with $\mathrm{GR}$ and the experiments.

\section{A constraint for the proper times of particles and a falling thin dust shell}

Let us consider a thin dust shell radially falling in own gravitational field. The metric outside and on the sphere $r \geq R$ is the Schwarzschild metric, the components of the interior metric are constant (homogeneous). For the particles of the shell freely falling from the rest at the spatial infinity, at a finite $r$ their coordinate velocity is given 
by $v(r)=d r / d t$. If at any large but finite radial coordinate $r_{1} \gg r_{g}$ we take zero initial times: $\tau_{1}=t_{1}=0$, then at $r<r_{1}$ we have the standard GR equation for the worldline $r(t)$ (in the reversed form):

$$
t=C\left(r_{1}\right)-2\left(r r_{g}\right)^{1 / 2}-\frac{2}{3} \frac{r^{3 / 2}}{r_{g}^{1 / 2}}+r_{g} \ln \left|\frac{r^{1 / 2}+r_{g}^{1 / 2}}{r^{1 / 2}-r_{g}^{1 / 2}}\right|,
$$

where $C\left(r_{1}\right)=$ const is chosen so that $t_{1}=0$. The same worldline, parameterized across the proper time, can be described also by the equation:

$$
\tau(r)=\frac{2}{3 r_{g}^{1 / 2}}\left(r_{1}^{3 / 2}-r^{3 / 2}\right), \quad r(\tau)=r_{1} \cdot\left(1-\tau / \alpha_{1}\right)^{2 / 3},
$$

where $\alpha_{1}=2 r_{1}^{3 / 2} / 3 r_{g}^{1 / 2}$.

Since two forms of the equations for the worldline (1) and (2) describe the same particle at the same point $r$ in terms of two kind of times, they relate any proper time moment $\tau[r(t)]$ with corresponding global time moment $t$ through the value $r(t)$ as a parameter. Therefore, the identity:

$$
r(t) \equiv r(\tau)
$$

is in fact a constraint $\tau=\tau(t)$ strongly relating the proper time moments of any event on the invariant worldline by the coordinate time moment. By inserting the value $r(\tau)$ from (2) into (1), we obtain the expression for the dependence $\tau(t)$ (Fig. 1) (also in the reversed form):

$$
t=C\left(r_{1}\right)-2\left(r_{1} r_{g}\right)^{1 / 2}\left(1-\frac{\tau}{\alpha_{1}}\right)^{1 / 3}-\alpha_{1}\left(1-\frac{\tau}{\alpha_{1}}\right)+r_{g} \ln \left|\frac{\left(1-\tau / \alpha_{1}\right)^{1 / 3}+\left(r_{g} / r_{1}\right)^{1 / 2}}{\left(1-\tau / \alpha_{1}\right)^{1 / 3}-\left(r_{g} / r_{1}\right)^{1 / 2}}\right| .
$$

The particles approach the gravitational radius $r \rightarrow r_{g}$ only at $t \rightarrow \infty$ while the corresponding proper time moment $\tau_{g}$ remains finite. But at any finite moment of world time $t<\infty$ we have $r(t)>r_{g}$ and the proper time $\tau[r(t)]$ of the particles will be less than $\tau_{g}$ required for the reaching $r_{g}$ :

$$
\begin{aligned}
& \tau_{g}-\tau(t)=\frac{2}{3 r_{g}^{1 / 2}}\left(r^{3 / 2}(t)-r_{g}^{3 / 2}\right)>0, \\
& \tau_{g}=\alpha_{1}\left[1-\left(r_{g} / r_{1}\right)^{3 / 2}\right] .
\end{aligned}
$$

At $r \rightarrow r_{g}$ one has approximately $r=r_{g}+h$, where $h \ll r_{g}$, and then the equations for the worldline and the relations between two times simplify:

$$
\begin{aligned}
& \tau \simeq \tau_{g}-h, \\
& t \simeq C^{\prime}+r_{g} \ln \left|\frac{2 r_{g}}{h}\right|=C^{\prime}+r_{g} \ln \left|\frac{2 r_{g}}{\tau_{g}-\tau}\right|,
\end{aligned}
$$


where $C^{\prime}=C\left(r_{1}\right)-8 r_{g} / 3$. By expressing $h$ as:

$$
h(t) \simeq \tau_{g}-\tau(t)=b \exp \left(-t / r_{g}\right)
$$

where $b=2 r_{g} \exp \left(C^{\prime} / r_{g}\right)$, we obtain an invariant constraint for $\tau(t)$ near $\tau_{g}$ :

$$
\tau(t)=\tau_{g}-b e^{-t / r_{g}}<\tau_{g} .
$$

Thus, at any $0 \leq t<\infty$ we will have $\tau(t)<\tau_{g}$, i.e. the constraint (4) or (8) restricts the proper time's rate by the world time rate on the global hypersurfaces of simultaneity (Fig.1). The worldline $r(\tau)$, apparently "cutted" near $r_{g}$, in fact is continuous and describes a full history of the particle for all moments of real world's time $t$. Previous attempts "to continue" this worldline to "interior" of $r_{g}$ have been related by misunderstanding of the physical foundations of general relativity.
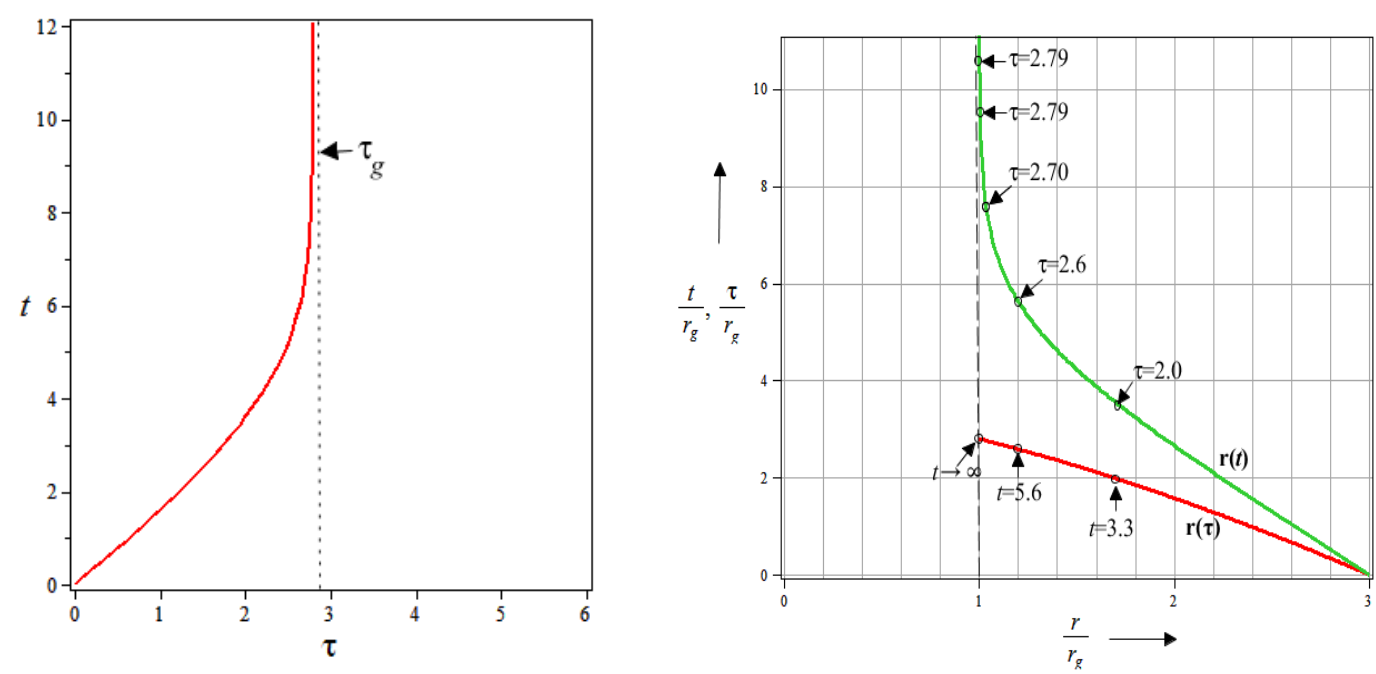

Fig.1. a) The constrained proper time $\tau=\tau(t)$ of a freely falling dust shell (left panel). The identity $r(t)=r(\tau)$ relates two kind of times and gives the invariant restriction $\tau=\tau_{g}-b \exp \left(-t / r_{g}\right)<\tau_{g}$ for large, but finite $t$ ( $\tau_{g}-$ dotted line). For a global set of synchronized coordinate clocks intervals $\Delta t$ are equidistant and local proper times are dilated $\tau(t)<t$. Initial values $t_{0}\left(r_{1}\right)=\tau_{0}\left(r_{1}\right)=0$ taken at $r_{1}=3 r_{g}$, times in units $r_{g} / c$. b) The same worldline of a dust shell's particle in two parametrizations - in world time $t$ and proper time $\tau$ (right panel). If $t$ is the ordinate, then along the worldline $\tau$ takes inequidistant values $\tau(t)$ - as close to $r_{g}$ its intervals become more and more stretched. If $\tau$ is the ordinate, then along the worldline $t$ takes inequdistant values $t(\tau)$ - as close to $r_{g}$ its intervals become more and more contracted. Due to the uniqueness and invariance of the particle's worldline, if it does not cross $r_{g}$ in one a parametrization, then it does not cross $r_{g}$ in other one too. The intervals of $\tau$ are equidistant only in local inertial frames near the particle and there is no a globally defined $\tau$ axis, so here $(\tau, r)$ plots are conditional and the physically correct ones are $(t, r)$ - plots only.

Since the latter (with insufficient corrections due to the local velocities) is nothing but as a mean evolution time rate of the Universe (in a rest frame of $\mathrm{CMB}$ ), 
and the intervals of $t$ are equidistant for most of the matter of the Universe, the proper times near the sources in fact are absolutely slowered with respect to this global evolution time of the Universe.

Thus, for any observer on the global hypersurface $t=$ const the dust shell does not cross own gravitational radius $\left(r(t)>r_{g}\right)$ and the transition to the proper times or any other parametrization does not change this invariant fact.

Since the surface of a sphere does not reach $r_{g}$ at any finite $t$, inside the shell, where the metric is homogeneous and the time dilation coefficient is the same as on the surface, the event horizon also does not arise.

Therefore, for the particles of non-zero mass the spacetime interval is timelike $d s^{2}>0$ everywhere $r \geq 0$, and the temporal and radial components of the metric remain regular both outside, on and inside the shell.

The same properties will have a system of enclosed spherical dust shells, and their free falling will not lead to the formation of the horizon neither outside, nor inside the such system of shells. Really, any shell changes the time dilation coefficient $g_{00}^{-1}\left(R_{n}\right)$ for all others to a finite value and at a finite mass it remains finite at any point: $1 \leq g_{00}^{-1}\left(R_{n}\right)<\infty$.

In the case of charged and rotating sources of the stationary gravitational field also there are two forms of equations of motion in terms of the world time or the proper time of falling test particles. These equations again give us a constraint for the proper times $\tau=\tau(t)$ so that at $t<\infty$ the surface will be outside the region where the time dilation coefficient tends to infinity.

\section{General properties of the gravitationally-frozen stars}

Contracting massive stars with surface at $R>r_{g}$ may be described in the first approximation as a set of enclosed dust shells of radius $r \leq R$. Let us consider general properties of the such stars becoming gravitationally-frozen at $R=r_{g}+h, h \ll r_{g}$ , due to large proper time dilation in entire volume, maximal at the centre and minimal on the surface. Their characteristic properties can be joined to the following groups [3]:

1. The large time dilation on the surface leads to the large redshift of a radiation emitted near the frozen surface. The radiated energy is concentrated on the long-wave region of the spectrum and the such stars should be powerful radio sources. The redshift of lines of massive stars, nuclei of galaxies and quasars, in addition to the cosmological redshift, will contain also the contribution of the gravitational redshift, and new methods for the separation of the contribution each of these mechanisms should be found.

2. Some of objects, catalogued as quasars, may by the Milky Way stars, the redshift of which has a purely gravitational origin.

3. Since for the extragalactic compact sources only a part of their redshift will have a gravitational origin, after the subtracting of that contribution, a pure cosmological redshift becomes less than the observing one. The such reducing of 
redshifts of high redshift quasars leads also to the reducing of their brightness, distance and mass.

4. The large time dilation near the surface also leads to the lack of sharp changes of parameters and very smooth, lengthened character of any peaks of intensity of the radiation. Particularly, this leads to a special character of the collisions of matter by the surface due to the time dilation in the form of a soft braking near the frozen surface.

5. Stationary compact objects do not create particles since world lines of particles are timelike and their energies are positive everywhere. Therefore the hypothetical quantum evaporation of spherical compact objects in GR is forbidden.

6. Non-stationary processes around these objects, having an external source for their energy, can lead to usual processes of pair creation. Thus, one of particles of a pair created at vicinity of the star can fall and will be frozen on the star, while other can leave the star. In terms of $t$ the leaving particles should be described via the density matrix containing mixed states of wave functions at different moments of proper times of quanta. The such partial loss of information because of the freezing a part of quantum fluctuations near the surface at the presence of a "thermostat" or external field, leads to the occurrence of an entropy and a temperature. Notice that this process leads not to the evaporation, but to growing of mass of the frozen star absorbing a part of energy of the external field or thermostat providing the such pair creation.

7. The frozen stars have higher local temperatures than the neutron stars, and, due to the interactions of practically adjoining hadrons, they become powerful sources of photons and neutrino. The energy loss due to various such effects, the bursts and explosions at internal phase transitions (also dilated in time), lead to slowly or rapidly "defrosting" of the frozen star.

8. The theory of quasars and galaxy nuclei, allowing one to understand their origin, now can be developed on a new fundamental basis. The gravitationally-frozen matter will be superdense mainly in compact central parts of quasars and AGNs. In the closer to surface layers the matter density may be ordinary, although may be highly frozen in terms of $t$. Therefore, the such objects have a complicated structure with matter in various phase states.

9. Some of "candidates for BHs" are more silent than the neutron stars and the thermonuclear explosions at falling of matter to the surface have not been observed. Some compact supermassive objects at the nucleus of some galaxies are silent also. These facts may confirm the new picture since the "explosions" and other locally fast phenomena become slow ones in terms of $t$. The such "delayed explosions", continuing hours and days, are really observed from time to time practically for all the such objects. Although the mechanisms of these phenomena may be complicated, the dilated explosions also probably contribute to the total effect. Moreover, the such dilations may be used for the measuring the time dilation coefficient near the surface of such compact objects.

10. New opportunities there will be opened by GR for the theories of supernovae explosions and for understanding of the nucleosynthesis which may occurs not only at explosions of the supernovae, but also during longer time in the gravitationally-frozen superdense stars by escaping out at the explosions due to the phase transitions. 


\section{Conclusion}

In GR the equations of motion and the identity $r(t) \equiv r(\tau)$ along worldlines constrains the proper time moments due to their dependence on the world time moments $\tau(t)$. As the result, at any $t<\infty$, we have $\tau(t)<\tau_{g}$, i.e. the constraint (4) - (8) restrict the proper time's moments by the world time moments on the globally defined hypersurfaces of simultaneity. This means that in the contracting star the proper times of falling particles become frozen so that at any finite coordinate time moment $t$ the proper time dilation is maximal at the centre and minimal on the surface of the star.

Thus, at any finite moment of the coordinate time GR predicts the gravitationally frozen superdense and supermassive stars with some internal structure where the spacetime interval for matter is timelike (lightlike for massless particles) and regular in entire space. The gravitationally-frozen matter is a new phase state of matter having new, unusual and in principle observable properties [3].

In contrast to the black holes, declared as fully isolated objects, the really predicting by GR frozen objects are observable at any finite moment of the coordinate time and may be sufficiently active. During the very slowered contraction the frozen star radiates (with high redshift), and at the internal phase transitions of matter with large energy liberation, there may be observed the bursts and explosions.

\section{References}

1. Oppenheimer J.R., Snyder H. (1939) Phys. Rev. 56, p. 455.

2. Misner C.W., Thorne K.S., Wheeler J.A. Gravitation. Freeman, 1973.

3. Zakir Z. (2006) Theor. Phys., Astrophys. \& Cosmol., 1, 3, 42; doi:

4. Haefele J., Keating R., Science 117 (1972) 168, 170.

5. Alley C. et al, Experim. Gravit., Proc. Conf. at Pavia (1976) N.Y., Acad. Press.

6. Okun L.B., Selivanov K.G., Telegdi V.L. (2000) Amer. J. Phys., 68, 115; arXiv:physics/9907017. 